\title{
Adaptive RAKE Combining Schemes in the Soft Handover Region*
}

\author{
A. B. A. B. Doua \\ Telecom Paris School (ENST) \\ Dept. of Communications and Electronics \\ Paris, France \\ benabdal@enst.fr
}

\author{
S. Choi, M.-S. Alouini, K. A. Qaraqe \\ Texas A\&M University at Qatar \\ Dept. of Electrical Engineering \\ Education City, Doha, Qatar \\ seyeong.choi, alouini, khalid.qaraqe@qatar.tamu.edu
}

\begin{abstract}
We propose and analyze new finger assignment schemes which are applicable for RAKE reception in the soft handover region. These schemes rely on variants of the conventional generalized selection combining (GSC) and the minimum selection GSC schemes in order to choose an acceptable base station and its corresponding paths. Relying on some recently derived results on order statistics, we investigate the average error rate performance as well as the average number of estimated and combined paths of the proposed schemes over independent and identically distributed Rayleigh fading channels. The mathematical formalism is illustrated with few selected numerical results that show the tradeoff between performance and processing power consumption.
\end{abstract}

\section{Introduction}

Multi-path fading is an unavoidable phenomena in wireless communications especially in wideband systems. Although the multi-path fading is one of the deteriorating factors in received signals, it can be exploited to improve the performance by using a RAKE receiver. The RAKE receiver is a technique which uses several baseband correlators called fingers to individually process multi-path signal components. The outputs from the different correlators are combined to achieve improved reliability and performance.

Considering that the different resolvable paths correspond to the diversity branches, we can use all diversity combining techniques for RAKE reception. For instance, with generalized selection combining (GSC) [1-5] which is a generalization of selection combining (SC), the receiver

* This work was funded in part by Qatar Telecom (Qtel) and in part by the Qatar Foundation for Education, Sceinces, and Community Development. chooses a fixed number of paths with the largest instantaneous signal-to-noise ratio (SNR) from all available diversity paths and then combines them as per the rules of maximal ratio combining (MRC). As a power-saving implementation of GSC, minimum selection GSC (MS GSC) [6-8] was recently proposed and studied. With MS GSC, after examining and ranking all available paths, the receiver tries to raise the combined SNR above a certain threshold by combining in an MRC fashion the least number of the best diversity paths, and as such, MS GSC can save considerable amount of processing power by keeping less MRC branch active on average in comparison to the conventional GSC scheme.

The aim of this work is to develop new variants of RAKE combing in the soft handover (SHO) region by applying the innovations of GSC and MS GSC schemes. While it is possible to use paths from different base stations (BSs) when the mobile is in the SHO region $[9,10]$, we rather propose in this paper several schemes which are designed to select paths from only one BS in order to minimize the use of network resources and facilitate the process of synchronization which tends to be complicated when the combined multi-paths come from different BSs. Through the mathematical analysis as well as numerical examples, we show that the proposed schemes save a considerable amount of complexity in terms of the average number of estimated and combined paths compared to the conventional GSC and MS GSC schemes but at the cost of an expected performance degradation that will be quantified in the body of this paper.

\section{System Model}

\subsection{System and Channel Model}

We assume that the RAKE combining schemes proposed in this work are implemented in a discrete-time fashion. More specifically, short guard periods are periodically inserted into the transmitted signal. During these guard periods, the receiver performs a series of operations, including 
path estimations and combined SNR comparisons with respect to the predetermined SNR threshold. Once the suitable paths and corresponding BS are selected, they are used throughout the subsequent data burst. Because of some hardware constraints, we assume that the receiver can only afford $L_{c}$ RAKE fingers, where $L_{c} \leq L$ and $L$ is the number of available paths from each BS. If we assume that there are $N$ BSs in the SHO region, among them the receiver chooses and uses only one BS whose resolvable paths are acceptable for data transmission based on the mode of operation that will be described in the next section.

Let $\gamma_{l}$ denote the instantaneous received SNR of the $l$ th resolvable path, $l=1,2, \cdots, L$. We assume slow frequency flat fading channels and we further adopt the block fading model where the fading coefficients are assumed to be constant through the data burst period. As such all the diversity paths experience almost the same fading conditions and maintain therefore the same SNR during the data burst and its preceding guard period. Moreover, the fading conditions are assumed to follow the Rayleigh model and to be i.i.d. across the diversity paths and between different guard periods and data bursts. As such, the faded SNR, $\gamma_{l}$, follows the same exponential distribution with the common average faded SNR, $\bar{\gamma}$.

\subsection{Mode of Operation}

When the conventional GSC scheme is used in the SHO region regardless of the origin of the combined paths, the receiver has to monitor simultaneously all the paths from all the BSs. To obtain desired savings in processing power and complexity of implementation in the mobile, we propose three different variants of the conventional GSC or MS GSC scheme. The proposed schemes are designed to just find and lock on the suitable resolvable paths of a certain BS.

2.2.1. Variant 1 - Based on Temporal Diversity. With this scheme, the receiver sequentially applies the MS GSC scheme to each BS. More specifically, after examining and ranking the $L$ paths of the currently used BS, the receiver raises the combined SNR above a certain threshold by combining in an MRC fashion the least number of the best diversity paths. If the $L_{c} / L$-MS GSC output SNR of the current BS is above the threshold, then the receiver continues to use it for data reception. On the other hand, if the combined output SNR of the best $L_{c}$ paths from this BS is still below the target threshold, the receiver tries to find an acceptable BS by sequentially examining in the same way the other $N-1$ BSs. This process is repeated until either an acceptable BS is found or all $N$ BSs have been examined. In the later case, since every output SNR of the $L_{c}$ best paths from each BS is known, the receiver uses the BS whose $L_{c} / L$-GSC output SNR is largest.
2.2.2. Variant 2 - Based on Space Diversity. Instead of using MS GSC on each BS sequentially as it was the case in the previous variant, the receiver estimates at first with this variant all $N L$ available paths from all BSs and compares the maximum of the strongest paths from each BS with the target SNR, i.e., $\max \left\{\gamma_{(1)}^{1}, \gamma_{(1)}^{2}, \cdots, \gamma_{(1)}^{N}\right\} \gtrless$ $\gamma_{T}$, where $\gamma_{(i)}^{k}$ is the $i$ th order statistics out of $L$ ones from the $k$ th BS such that $\gamma_{(1)}^{k} \geq \gamma_{(2)}^{k} \geq \cdots \geq \gamma_{(L)}^{k}$. If $\max \left\{\gamma_{(1)}^{1}, \gamma_{(1)}^{2}, \cdots, \gamma_{(1)}^{N}\right\} \geq \gamma_{T}$, then this strongest path and its corresponding BS will be used for data reception. Otherwise, the tested combined SNR becomes the maximum of the sums of two strongest paths, i.e., $\max \left\{\Gamma_{2}^{1}, \Gamma_{2}^{2}, \cdots, \Gamma_{2}^{N}\right\} \gtrless \gamma_{T}$, where $\Gamma_{i}^{k}\left(=\sum_{a=1}^{i} \gamma_{(a)}^{k}\right)$ is the partial sum of the first $i$ order statistics. By adding the next strongest paths, this process is repeated until either the acceptable best partial sum is found or all of these partial sums are less than $\gamma_{T}$, i.e., $\max \left\{\Gamma_{L_{c}}^{1}, \Gamma_{L_{c}}^{2}, \cdots, \Gamma_{L_{c}}^{N}\right\}<$ $\gamma_{T}$. In the later case, similar to Variant 1 , the receiver chooses the BS whose $L_{c} / L$-GSC output SNR is the largest.

2.2.2. Variant 3 - Mixture of Variants 1 and 2. This scheme works as a combinational form of Variants 1 and 2. The receiver starts to use Variant 1 with $1 / L$-MS GSC to find the acceptable strongest path. More specifically, the receiver selects the strongest path of the current BS, $\gamma_{(1)}^{1}$, and switches to the strongest path of the second BS, $\gamma_{(1)}^{2}$, if $\gamma_{(1)}^{1}<\gamma_{T}$. This process is continued up to the $N$ th BS. If it turns out that the strongest path of all $N$ BSs are below the target SNR, then the receiver applies Variant 2 by starting to check the sum of two strongest paths, i.e., $\max \left\{\Gamma_{2}^{1}, \Gamma_{2}^{2}, \cdots, \Gamma_{2}^{N}\right\} \gtrless \gamma_{T}$, since all $N L$ available paths have already been estimated.

\section{Statistics of Combined SNR}

\subsection{Variant 1}

With this Variant, the receiver applies the MS GSC mode of operation to one BS and switches to a new one if this BS is unacceptable. Therefore, if we let $\gamma$ denote the final combined SNR, then the cumulative distribution function (CDF) of $\gamma, F_{\gamma}(x)$, can be expressed as

$$
\begin{aligned}
& F_{\gamma}(x)=\operatorname{Pr}[\gamma<x] \\
= & \begin{cases}\operatorname{Pr}\left[\gamma_{M S}^{1} \geq \gamma_{T}, \gamma_{M S}^{1}<x\right] \\
+\sum_{k=2}^{N} \operatorname{Pr}\left[\Gamma_{L_{c}}^{1}<\gamma_{T}, \Gamma_{L_{c}}^{2}<\gamma_{T},\right. \\
\left.\cdots, \Gamma_{L_{c}}^{k-1}<\gamma_{T}, \gamma_{M S}^{k} \geq \gamma_{T}, \gamma_{M S}^{k}<x\right], & x \geq \gamma_{T} ; \\
\operatorname{Pr}\left[\max \left\{\Gamma_{L_{c}}^{1}, \Gamma_{L_{c}}^{2}, \cdots, \Gamma_{L_{c}}^{N}\right\}<x\right], & x<\gamma_{T},\end{cases}
\end{aligned}
$$


where $\gamma_{M S}^{k}$ is the $L_{c} / L$-MS GSC output SNR from the $k$ th BS. Since we assume an equal number of i.i.d. available paths from each BS, we can ignore the superscripts indicating the BSs. Hence, some manipulations of (1) give the $\mathrm{CDF}$ of $\gamma$ as

$$
F_{\gamma}(x)= \begin{cases}\frac{1-\left[F_{\Gamma_{L_{c}}}\left(\gamma_{T}\right)\right]^{N}}{1-F_{\Gamma_{L_{c}}}\left(\gamma_{T}\right)} & \\ \times\left[F_{\gamma_{M S}}(x)-F_{\gamma_{M S}}\left(\gamma_{T}\right)\right], & x \geq \gamma_{T} ; \\ {\left[F_{\Gamma_{L_{c}}}(x)\right]^{N},} & x<\gamma_{T},\end{cases}
$$

where $F_{\gamma_{M S}}(\cdot)$ and $F_{\Gamma_{L_{c}}}(\cdot)$ are the CDFs of $L_{c} / L$-MS GSC and $L_{c} / L$-GSC output SNRs which can be found in [8, Eq. (20)] and [3, Eq. (24)], respectively. Differentiation of (2) with respect to $x$ gives the probability density function (PDF) of $\gamma, f_{\gamma}(x)$, as

$$
f_{\gamma}(x)= \begin{cases}\frac{1-\left[F_{\Gamma_{L_{c}}}\left(\gamma_{T}\right)\right]^{N}}{1-F_{\Gamma_{L_{c}}}\left(\gamma_{T}\right)} f_{\gamma_{M S}}(x), & x \geq \gamma_{T} ; \\ N\left[F_{\Gamma_{L_{c}}}(x)\right]^{N-1} f_{\Gamma_{L_{c}}}(x), & x<\gamma_{T},\end{cases}
$$

where $f_{\gamma_{M S}}(\cdot)$ and $f_{\Gamma_{L_{c}}}(\cdot)$ are the PDFs of $L_{c} / L$-MS GSC and $L_{c} / L$-GSC output SNRs which can be found in [8, Eq. (34)] and [3, Eq. (16)], respectively.

\subsection{Variant 2}

Note that this scheme selects the BS whose sum of the $i$ strongest paths is above the threshold, $\gamma_{T}$, starting from $i=1$ to $L_{c}$. To simplify the presentation, if we let $\Gamma_{i}^{*}$ denote the maximum partial sum of $i$ strongest paths from each BS such as

$$
\Gamma_{i}^{*}=\max \left\{\Gamma_{i}^{1}, \Gamma_{i}^{2}, \cdots, \Gamma_{i}^{N}\right\},
$$

then applying the total probability theorem, we can write the CDF of $\gamma$ as

$$
\begin{aligned}
& F_{\gamma}(x)=\operatorname{Pr}[\gamma<x] \\
= & \begin{cases}\operatorname{Pr}\left[\gamma_{T} \leq \Gamma_{1}^{*}<x\right] \\
+\sum_{i=2}^{L_{c}} \operatorname{Pr}\left[\Gamma_{i-1}^{*}<\gamma_{T}, \gamma_{T} \leq \Gamma_{i}^{*}<x\right], & x \geq \gamma_{T} ; \\
\operatorname{Pr}\left[\max \left\{\Gamma_{L_{c}}^{1}, \Gamma_{L_{c}}^{2}, \cdots, \Gamma_{L_{c}}^{N}\right\}<x\right], & x<\gamma_{T} .\end{cases}
\end{aligned}
$$

With the following relationships

$$
\operatorname{Pr}\left[\gamma_{T} \leq \Gamma_{1}^{*}<x\right]=\left[F_{\Gamma_{1}}(x)\right]^{N}-\left[F_{\Gamma_{1}}\left(\gamma_{T}\right)\right]^{N}
$$

and

$$
\begin{aligned}
& \operatorname{Pr}\left[\Gamma_{i-1}^{*}<\gamma_{T}, \gamma_{T} \leq \Gamma_{i}^{*}<x\right] \\
& =\left[F_{\Gamma_{i}, \Gamma_{i-1}}\left(x, \gamma_{T}\right)\right]^{N}-\left[F_{\Gamma_{i}, \Gamma_{i-1}}\left(\gamma_{T}, \gamma_{T}\right)\right]^{N},
\end{aligned}
$$

where $F_{\Gamma_{i}, \Gamma_{i-1}}(\cdot, \cdot)$ is the joint CDF of two partial sums of ordered SNRs, $\Gamma_{i}$ and $\Gamma_{i-1}$, differentiation of (5) gives the
PDF of $\gamma$ as

$$
f_{\gamma}(x)= \begin{cases}N\left[F_{\Gamma_{1}}(x)\right]^{N-1} f_{\Gamma_{1}}(x) & \\ +\sum_{i=2}^{L_{c}} N\left[F_{\Gamma_{i}, \Gamma_{i-1}}\left(x, \gamma_{T}\right)\right]^{N-1} & \\ \times \frac{d}{d x} F_{\Gamma_{i}, \Gamma_{i-1}}\left(x, \gamma_{T}\right), & x \geq \gamma_{T} ; \\ N\left[F_{\Gamma_{L_{c}}}(x)\right]^{N-1} f_{\Gamma_{L_{c}}}(x), & x<\gamma_{T} .\end{cases}
$$

The closed-form expression for the joint CDF, $F_{\Gamma_{i}, \Gamma_{i-1}}(x, y)$, in (8) can be found in [11, Eq, (33)] and its derivative, $\frac{d}{d x} F_{\Gamma_{i}, \Gamma_{i-1}}\left(x, \gamma_{T}\right)$, can be also obtained in closed-form since

$$
\begin{aligned}
\frac{d}{d x} F_{\Gamma_{i}, \Gamma_{i-1}}\left(x, \gamma_{T}\right) & =\int_{0}^{\gamma_{T}} f_{\Gamma_{i}, \Gamma_{i-1}}(x, y) d y \\
& =\int_{x \frac{i-1}{i}}^{\gamma_{T}} f_{\gamma_{(i)}, \Gamma_{i-1}}(x-y, y) d y,
\end{aligned}
$$

where the exact closed-form expression for the last term in (9) is given in [8, Eq. (32)].

\subsection{Variant 3}

Note that this scheme starts with Variant 1 to find the acceptable strongest path and then works as Variant 2. Hence, based on this mode of operation, it can be easily shown that the PDF of $\gamma$ is a combination of (3) and (8) given by

$$
f_{\gamma}(x)=\left\{\begin{array}{lr}
\frac{1-\left[F_{\Gamma_{1}}\left(\gamma_{T}\right)\right]^{N}}{1-F_{\Gamma_{1}}\left(\gamma_{T}\right)} f_{\Gamma_{1}}(x) & \\
+\sum_{i=2}^{L_{c}} N\left[F_{\Gamma_{i}, \Gamma_{i-1}}\left(x, \gamma_{T}\right)\right]^{N-1} & (10) \\
\times \frac{d}{d x} F_{\Gamma_{i}, \Gamma_{i-1}}\left(x, \gamma_{T}\right), & x \geq \gamma_{T} \\
N\left[F_{\Gamma_{L_{c}}}(x)\right]^{N-1} f_{\Gamma_{L_{c}}}(x), & x<\gamma_{T} .
\end{array}\right.
$$

\section{Performance Analysis ${ }^{1}$}

\subsection{Average Bit Error Rate}

We apply the results from the previous section to the well-known PDF-based approach [8, Eq. (31)] in order to obtain the average bit error rate (BER) of our proposed combining schemes over i.i.d. Rayleigh fading channels. In Fig. 1, we compare the average BER of binary phase shift keying (BPSK) versus (a) the average SNR per path, $\bar{\gamma}$, with $\gamma_{T}=5 \mathrm{~dB}$ and (b) the output threshold, $\gamma_{T}$, with $\bar{\gamma}=0 \mathrm{~dB}$ when used in conjunction with the proposed schemes over i.i.d. Rayleigh fading channels when $N=4, L=6$, and $L_{c}=4$. For comparison purpose, we also plot the average BER of the conventional $L_{c} / N L$-GSC and $L_{c} / N L$-MS GSC. As shown in the statistics derived in the previous section, when the channel quality is poor, i.e., low average SNR

\footnotetext{
${ }^{1}$ As a double check, all numerical evaluations obtained from the analytical results derived in this paper have been compared and verified by Monte Carlo simulations.
} 


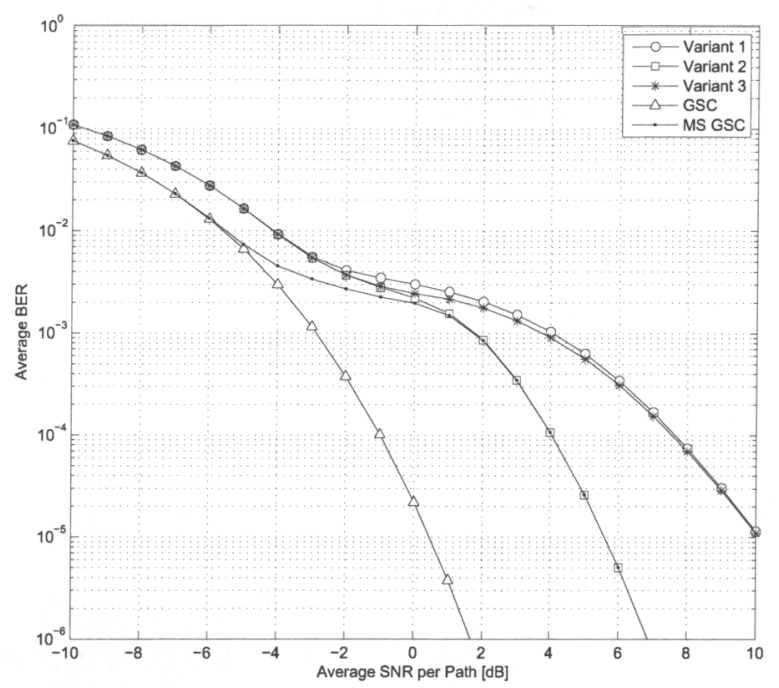

(a) Average BER versus $\bar{\gamma}$ when $\gamma_{T}=5 \mathrm{~dB}$

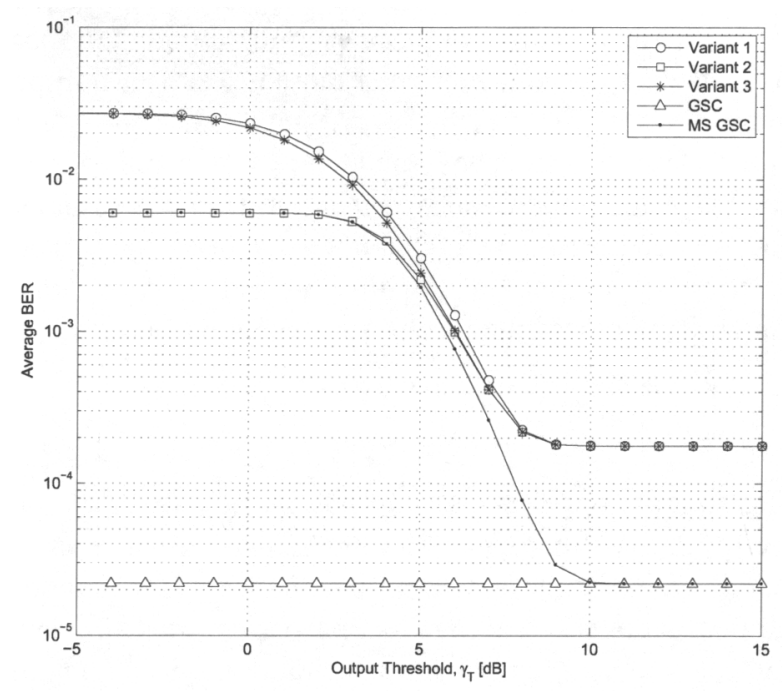

(b) Average BER versus $\gamma_{T}$ when $\bar{\gamma}=0 \mathrm{~dB}$

Figure 1. Average BER of the proposed schemes, conventional GSC, and conventional MS GSC over i.i.d. Rayleigh fading channels when $N=4, L=6$, and $L_{c}=4$.

region in Fig. 1 (a) and high threshold region in Fig. 1 (b), all proposed schemes show the same performance. However, as the channel quality improves, we can observe a certain performance gap between the different variants. More specifically, in this latter case, Variant 2 acts as MS GSC while Variants 1 and 3 are quite outperformed. This arises with Variants 1 and 3 because when the channel conditions are good, there is a high probability that the strongest path of the first scanned BS is good enough to reach the target SNR while for Variant 2, the receiver always seeks the best strongest path from all BSs. Hence, we can expect that Variant 2 is performing some unnecessary path estimations compared to Variants 1 and 3, but by doing so reduces the number of combined paths. We investigate in what follows this tradeoff by exactly quantifying the average number of estimated and combined paths of the proposed schemes.

\subsection{Average Number of Estimated and Combined Paths}

From channel estimation complexity and processing power consumption perspectives, it is of interest to study the statistics of the number of estimated paths, $N_{E}$, and combined paths, $N_{C}$, per data burst. Note that both $N_{E}$ and $N_{C}$ are discrete random variables (RVs) with a certain probability mass function (PMF) that we will calculate for each variant. We then deduce the average number of estimated paths, $\bar{N}_{E}$, as well as the average number of combined paths, $\bar{N}_{C}$, per data burst.

The average number of estimated paths can be calculated as

$$
\bar{N}_{E}=\sum_{k=1}^{N} k L \operatorname{Pr}\left[N_{E}=k L\right],
$$

where $\operatorname{Pr}\left[N_{E}=k L\right]$ is the PMF of $N_{E}$. Based on the mode of operation of each variant, we can express the PMFs of Variants 1 and 3 as

$$
\begin{aligned}
& \operatorname{Pr}\left[N_{E}^{\operatorname{Var} 1}=k L\right] \\
& = \begin{cases}1-F_{\Gamma_{L_{c}}}\left(\gamma_{T}\right), & k=1 ; \\
{\left[F_{\Gamma_{L_{c}}}\left(\gamma_{T}\right)\right]^{k-1}\left[1-F_{\Gamma_{L_{c}}}\left(\gamma_{T}\right)\right],} & 1<k<N ; \\
{\left[F_{\Gamma_{L_{c}}}\left(\gamma_{T}\right)\right]^{N-1},} & k=N\end{cases}
\end{aligned}
$$

and

$$
\begin{aligned}
& \operatorname{Pr}\left[N_{E}^{\operatorname{Var} 3}=k L\right] \\
& = \begin{cases}1-F_{\Gamma_{1}}\left(\gamma_{T}\right), & k=1 ; \\
{\left[F_{\Gamma_{1}}\left(\gamma_{T}\right)\right]^{k-1}\left[1-F_{\Gamma_{1}}\left(\gamma_{T}\right)\right],} & 1<k<N ; \\
{\left[F_{\Gamma_{1}}\left(\gamma_{T}\right)\right]^{N-1},} & k=N,\end{cases}
\end{aligned}
$$

respectively, while the number of estimated paths of Variant 2 is deterministic and equal to $N L$ since at first all paths are estimated in order to take the best strongest path among all paths.

The average number of combined paths is a more interesting metric for the system designers because less number of combined paths leads to considerable saving in mobile 
Table 1. Average number of estimated paths and combined paths of the proposed schemes, conventional GSC, and conventional MS GSC.

\begin{tabular}{|c|c|c|}
\hline Variants & $\bar{N}_{E}$ & $\bar{N}_{C}$ \\
\hline \hline V1 & $L \frac{1-\left[F_{\Gamma_{L_{c}}}\left(\gamma_{T}\right)\right]^{N}}{1-F_{\Gamma_{c}}\left(\gamma_{T}\right)}$ & $\left(1+\sum_{l=1}^{L_{c}-1} F_{\Gamma_{l}}\left(\gamma_{T}\right)\right) \frac{1-\left[F_{\Gamma_{c}}\left(\gamma_{T}\right)\right]^{N}}{1-F_{\Gamma_{L}}\left(\gamma_{T}\right)}+L_{c} \frac{\left[F_{\Gamma_{L_{c}}}\left(\gamma_{T}\right)\right]^{N}-F_{\Gamma_{L_{c}}}\left(\gamma_{T}\right)}{1-F_{\Gamma_{L_{c}}}\left(\gamma_{T}\right)}$ \\
\hline V2 & $N L$ & $1+\sum_{l=1}^{L_{c}-1}\left[F_{\Gamma_{l}}\left(\gamma_{T}\right)\right]^{N}$ \\
\hline V3 & $L \frac{1-F_{\Gamma_{1}}\left(\gamma_{T}\right)^{N}}{1-F_{\Gamma_{1}}\left(\gamma_{T}\right)}$ & $1+\sum_{l=1}^{L_{c}-1}\left[F_{\Gamma_{l}}\left(\gamma_{T}\right)\right]^{N}$ \\
\hline GSC & $N L$ & $L_{c}$ \\
\hline MS GSC & $N L$ & $1+\sum_{l=1}^{L_{c}-1} P_{\Gamma_{l}}\left(\gamma_{T}\right)$, where $P_{\Gamma_{l}}\left(\gamma_{T}\right)$ is the CDF of $l / N L$-GSC \\
\hline
\end{tabular}

receiver processing power. It can be obtained by

$$
\bar{N}_{C}=\sum_{l=1}^{L_{c}} l \operatorname{Pr}\left[N_{C}=l\right]
$$

where $\operatorname{Pr}\left[N_{C}=l\right]$ is the PMF of $N_{C}$. Following the mode of operation of Variants 1, 2, and 3, we have

$$
\begin{aligned}
\operatorname{Pr} & {\left[N_{C}^{V a r 1}=l\right] } \\
= & \begin{cases}{\left[1-F_{\Gamma_{1}}\left(\gamma_{T}\right)\right] \frac{1-\left[F_{\Gamma_{L_{c}}}\left(\gamma_{T}\right)\right]^{N}}{1-F_{\Gamma_{c}}\left(\gamma_{T}\right)},} & l=1 \\
{\left[F_{\Gamma_{l-1}}\left(\gamma_{T}\right)-F_{\Gamma_{l}}\left(\gamma_{T}\right)\right]} & 1-\left[F_{\Gamma_{L_{c}}}\left(\gamma_{T}\right)\right]^{N} \\
\times \frac{1-F_{\Gamma_{c}}\left(\gamma_{T}\right)}{\left.1 F_{\Gamma_{L_{c}-1}}\left(\gamma_{T}\right)-F_{\Gamma_{L_{c}}}\left(\gamma_{T}\right)\right]} & 1<l<L_{c} \\
\times \frac{1-\left[F_{\Gamma_{c}}\left(\gamma_{T}\right)\right]^{N}}{1-F_{\Gamma_{L_{c}}}\left(\gamma_{T}\right)}+\left[F_{\Gamma_{L_{c}}}\left(\gamma_{T}\right)\right]^{N}, & l=L_{c}\end{cases}
\end{aligned}
$$

and

$$
\begin{aligned}
\operatorname{Pr}\left[N_{C}^{V a r 2}=l\right] & =\operatorname{Pr}\left[N_{C}^{V a r 3}=l\right] \\
& = \begin{cases}1-\left[F_{\Gamma_{1}}\left(\gamma_{T}\right)\right]^{N}, & l=1 ; \\
{\left[F_{\Gamma_{l-1}}\left(\gamma_{T}\right)\right]^{N}} & \\
-\left[F_{\Gamma_{l}}\left(\gamma_{T}\right)\right]^{N}, & 1<l<L_{c} \\
{\left[F_{\Gamma_{L c-1}}\left(\gamma_{T}\right)\right]^{N},} & l=L_{c} .\end{cases}
\end{aligned}
$$

Substituting (12) and (13) into (11), and (15) and (16) into (14), we can obtain $\bar{N}_{E}$ and $\bar{N}_{C}$ for all variants which are summarized in Table 1 along those of conventional GSC and MS GSC [8].

In Fig. 2, we plot (a) the average number of estimated paths and (b) the average number of combined paths versus the output threshold, $\gamma_{T}$, of the proposed schemes and the conventional $L_{c} / N L$-GSC and $L_{c} / N L$-MS GSC schemes over i.i.d. Rayleigh fading channels when $N=4, L=$ $6, L_{c}=4$, and $\bar{\gamma}=0 \mathrm{~dB}$. Note that with the conventional GSC and MS GSC schemes, the paths are combined regardless of BSs while our proposed schemes select paths from only one BS. In terms of the estimation load, GSC, MS GSC, and Variant 2 have the highest constant value of $\bar{N}_{E}=N L$ since these schemes always have to select the best path among all available paths while Variants 1 and 3 estimate less paths especially when the channel quality is good because of their sequential scanning initialization. Note that Variant 3 works as Variant 1 in the relatively high average SNR region and as Variant 2 in the low average SNR region, which overall results in a higher estimation load than Variant 1, as illustrated in Fig. 2 (a).

For the average number of combined paths, MS GSC shows the minimum number of combined paths while GSC has the highest constant value of $\bar{N}_{C}=L_{c}$. Unlike the estimation load in Fig. 2 (a), Variant 1 requires more combined paths than the other schemes, and this illustrates well the tradeoff between the estimation load and the combined paths load. Also note that Variants 2 and 3 have the same $\bar{N}_{C}$ and their values move from that of MS GSC to that of Variant 1 as $\gamma_{T}$ increases.

Observation of Fig. 1 together with Fig. 2 shows that when the channel quality is relatively good compared to the threshold, Variant 2 has the better performance at the sacrifice of the path estimation load. On the other hand, in a poor channel quality, since all the variants show almost the same performance, choosing Variant 1 benefits from the less estimation load while choosing Variants 2 or 3 benefits from the reduction of processing power.

\section{Conclusion}

In this paper, we proposed new finger assignment schemes for RAKE receivers in the SHO region. With these schemes, a proper BS is selected by the receiver and three different selection algorithms based on the variants of the conventional GSC and MS GSC schemes are proposed. We derived the statistics of the output SNR of the proposed schemes in accurate closed-form, based on which we carried out the performance analysis of the resulting sys- 


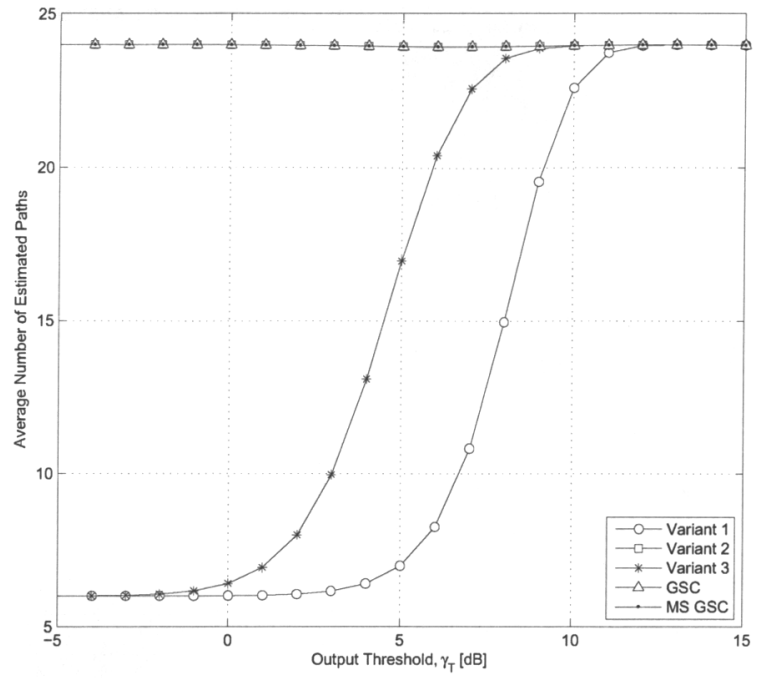

(a) Average number of estimated paths

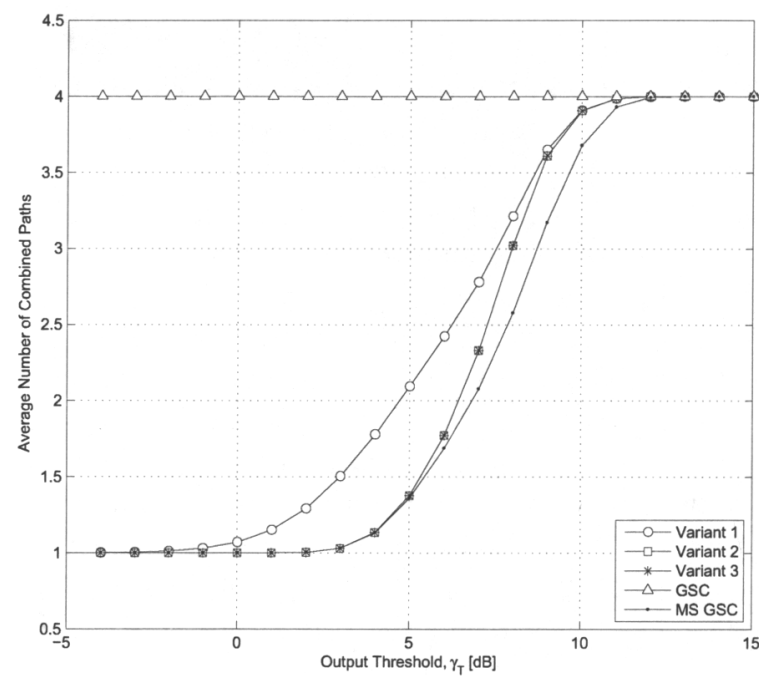

(b) Average number of combined paths

Figure 2. Average number of estimated paths and combined paths of the proposed schemes, conventional GSC, and conventional MS GSC versus the output threshold, $\gamma_{T}$, over i.i.d. Rayleigh fading channels when $N=4, L=6, L_{c}=4$, and $\bar{\gamma}=0 \mathbf{d B}$.
Through some selected numerical examples, the tradeoff between performance and processing power consumption has been investigated.

\section{References}

[1] T. Eng, N. Kong, and L. B. Milstein, "Comparison of diversity combining techniques for Rayleigh-fading channels," IEEE Trans. Commun., vol. 44, no. 9, pp. 1117-1129, Sept. 1996.

[2] M. Z. Win and J. H. Winters, "Analysis of hybrid selection/maximal-ratio combining in Rayleigh fading," IEEE Trans. Commun., vol. 47, no. 12, pp. 1773-1776, Dec. 1999.

[3] M.-S. Alouini and M. K. Simon, "An MGF-based performance analysis of generalized selection combining over Rayleigh fading channels," IEEE Trans. Commun., vol. 48, no. 3, pp. 401-415, Mar. 2000.

[4] Y. Ma and C. C. Chai, "Unified error probability analysis for generalized selection combining in Nakagami fading channels," IEEE J. Select. Areas Commun., vol. 18, no. 11, pp. 2198-2210, Nov. 2000.

[5] A. Annamalai and C. Tellambura, "Analysis of hybrid selection/maximal-ratio diversity combiner with Gaussian errors," IEEE Trans. Wireless Commun., vol. TWC-1, no. 3, pp. 498-512, July 2002.

[6] S. W. Kim, D. S. Ha, and J. H. Reed, "Minimum selection GSC and adaptive low-power RAKE combining scheme," in Proc. IEEE Int. Symp. on Circuit and Systems (ISCAS'03), Bangkok, Thailand, May 2003.

[7] R. K. Mallik, P. Gupta, and Q. T. Zhang, "Minimum selection GSC in independent Rayleigh fading," IEEE Trans. Veh. Technol., vol. 54, no. 3, pp. 1013-1021, May 2005.

[8] H.-C. Yang, "New results on ordered statistics and analysis of minimum-selection generalized selection combining (GSC)," IEEE Trans. Wireless Commun., vol. 5, no. 7, pp. 1876-1885, July 2006.

[9] S. Choi, M.-S. Alouini, K. A. Qaraqe, and H.-C. Yang, "Finger assignment schemes for RAKE receivers with multi-way soft handover," in Proc. IEEE Wireless Commun. \& Networking Conf. (WCNC'07), Hong Kong, Mar. 2007.

[10] _ - "Finger replacement schemes for RAKE receptions in the soft handover region with multiple base stations," in Proc. IEEE Int. Symp. on Personal, Indoor and Mobile Radio Comm. (PIMRC'07), Athens, Greece, Sept. 2007.

[11] Z. Bouida, N. Belhaj, M.-S. Alouini, and K. A. Qaraqe, "Minimum selection GSC with down link power control," IEEE Trans. Wireless Commun., To appear.

tems. More specifically, we considered the average error rate performance as well as the average number of estimated and combined paths of the proposed schemes over independent and identically distributed Rayleigh fading channels. 